\title{
Selective access in cued recall: The roles of retrieval cues and domains of encoding
}

\author{
CATHY L. MCEVOY and DOUGLAS L. NELSON \\ University of South Florida, Tampa, Florida
}

\begin{abstract}
The purpose of these experiments was to determine whether retrieval access in cued recall can be selectively restricted to a single domain of encoding when information has been encoded within two domains as different as word sound and word meaning. In Experiment 1, the subjects studied pairs of rhyming words differing in rhyme set size under verbal-repetition or interactive-imagery instructions. Recall was cued with study-context rhymes, with extralist rhymes, or with extralist associates. The results indicated that rhyme set size and instructional effects were found no matter how recall was cued, indicating that both domains of encoding were always accessed. In Experiment 2, potential effects of study time and overt naming of the test cues were explored. These results indicated that both domains of encoding were accessed, except when long study times were available and subjects did not have to name the rhyme test cues. Rhyme set-size effects were eliminated under these conditions, suggesting that selective access to encoded meaning is possible in cued recall. Retrieval access in this task appears to be more controlled by domains of encoding than by information directly available in the cue, and access to encoded information can be restricted to a single domain.
\end{abstract}

William James (1890/1962) suggested that "we make search in our memory for a forgotten idea, just as we rummage our house for a lost object. In both cases, we visit what seems to us the probable neighborhood [italics added] of that which we miss" (p. 297). This conception assumes that retrieval is not haphazard but is directed toward specific knowledge. This idea has been incorporated into modern conceptions with the assumption that retrieval is a goaldirected process involving controlled access to particular domains of knowledge (e.g., Fisher \& Craik, 1977; Nelson, 1989; Raaijmakers \& Shiffrin, 1981; Shiffrin, 1970). These domains are defined in part by information residing in the retrieval context. This context often involves a conjunction of instructions, general environmental cues, and specific cues that serve the self-addressing function of indicating the probable neighborhood of the information sought. For example, access to a recently encoded familiar word can be obtained through cues that specify its speech code or through cues that specify its meaning (Nelson, 1989). In the one instance, the cue defines a probable neighborhood of lexically related concepts, and in the other, a probable neighborhood of meaningfully related concepts.

The effectiveness of such cues as aids to recall is usually attributed to activation of associative links between the cues and their targets (Anderson, 1983; Bahrick, 1970) and to reductions in the size of the probable neighborhood (Anderson, 1983; Nelson, 1981, 1989). Such attri-

This research was supported by Grant MH 16360 from the National Institute of Mental Health to Douglas L. Nelson. Many thanks go to Elaine Georgilas for collecting the data for Experiment 2. Correspondence concerning this article should be addressed to Cathy L. McEvoy, Department of Aging and Mental Health, Florida Mental Health Institute, Tampa, FL 33612.

-Accepted by previous editor, Alice F. Healy butions could be interpreted as implying that access is confined to the domain of information represented in the retrieval cue: Meaning cues access meaningfully related concepts and rhyme cues access lexically related concepts. However, available findings indicate that access is not uniformly confined to the domain of information prescribed by specific cues. Meaning cues can be used to recover studied words (targets) involved in rhyme encodings, and rhyme cues can be used to recover targets involved in semantic encodings (Fisher \& Craik, 1977; Nelson, Walling, \& McEvoy, 1979). Furthermore, when subjects must switch randomly between rhyme and meaning cues at test, they sometimes recall targets from the other domain (Nelson, McEvoy, \& Friedrich, 1982). This interference occurs even though the correct domain is indicated for each cue (e.g., the cue BOY [rhyme] leads to the recall of MALE instead of TOY, and BOY [meaning] leads to the recall of TOY instead of MALE).

These results indicate that cues that provide information in only a single domain of information can be used to recover information encoded in another domain, and, at least under some conditions, subjects access the incorrect domain even when the domains are as disparate as rhyme and meaning. Related findings indicate that when information has been encoded within two different domains, both domains are accessed at test. These findings are based on set-size effects (Nelson, 1981, 1989). For a given word, rhyme set size refers to the number of other English words sharing its word stem, and meaning set size refers to the number of its meaningfully related associates. Words with larger sets of either type are not as likely to be recalled in the presence of cues as are those with smaller sets. This set-size effect is attributed to search processes that occur during retrieval (Nelson, 1981, 
1989). Rhyme set-size effects are contingent on the drawing of attention to word sound during study or test (Nelson, Bajo, \& Canas, 1987). Meaning set-size effects are found when the study context fails to specify or modify the meaning of the target, as when a studied word is presented in the absence of a specific context word (Nelson \& Friedrich, 1980).

Most importantly, both rhyme and meaning set-size effects are independent of the domain of information specified in the test cue. Provided that rhyme is emphasized during study (as when CLOCK BLOCK is studied), rhyme set-size effects are apparent with the original context word (CLOCK) and with extralist rhyme (SHOCK) and meaning (BRICK) cues. Provided that meaning is not specified at study by the context, meaning set-size effects are also obtained with both extralist rhyme and extralist meaning cues. According to the sensory-semantic model, the presentation of a familiar word in the absence of contextual cues that modify its meaning automatically activates its associates (Nelson, 1989; Nelson, Bajo, \& Canas, 1987). For example, the presentation of the word BLOCK theoretically activates its representation, which in turn activates associates such as BRICK, CEMENT, and WOOD. Furthermore, provided that subjects attend to rhyme, the presentation of the word BLOCK in a rhyme context activates related rhymes such as SHOCK and ROCK. According to the model, subjects habitually attend to meaning and they attend to rhyme when instructed to do so. Although the initial orientation to the two domains differs, once attention is directed to a particular domain, concepts related to the presented target appear to be automatically activated within the attended domain (Nelson, Bajo, \& Canas, 1987).

The activation of these concepts theoretically incorporates them into the episodic encoding. They interfere at test when concepts activated by the cue in one domain are used to recover the episodic encoding (Nelson et al., 1982). For example, as test cues, rhymes activate related rhymes, and these entries serve as internal cues for recovering information encoded about both rhyme and meaning. Similarly, meaning cues activate their associates and these associates serve as cues for accessing information encoded about rhyme and meaning. In the cued recall task, the activation of information in one domain is used to recover information encoded in both domains. In this manner both rhyme and meaning target set-size effects are obtained with cues from either domain. However, it is largely unresolved whether these entries always activate information in both domains of the episodic encoding or whether they can be used to provide selective access to these domains.

The purpose of the present study was to determine whether retrieval access to information encoded in two different domains, rhyme and meaning, can be selectively controlled. To the extent that access can come under selective control, it should be possible to access information in a single domain even when information has been en- coded in two domains. Alternatively, if access is not selective, all domains of encoding should invariably be accessed, regardless of the domains involved, the cues used, and other testing requirements. One way to distinguish between these alternatives is to isolate conditions in which encoding occurs within two domains with access limited to a single domain, a result that would be indicative of selective access. Such conditions may not have been met in earlier experiments involving set-size manipulations because of the nature of the encoded information being retrieved. The independence of set-size effects and type of test cue is based on encodings that appear to involve automatically activated information at study. Such independence may not generalize to encodings linked to more effortful or elaborative processing. With elaborative processing at study, access may be more selective at test. For example, semantic encodings resulting from interactive imagery may be more likely to be bypassed when testing is prompted by rhyme cues.

The specific purpose of these experiments was to explore the question of selective domain access at test when the episodic semantic encoding involved elaborative processing in addition to the automatic activation of related concepts. In Experiment 1, one group of subjects studied pairs of rhyming words using verbal-repetition instructions and another group studied the same pairs using interactive-imagery instructions. Both groups were expected to encode the pairs semantically as well as phonemically, but imagery instructions were expected to result in more elaborate semantic encodings than were repetition instructions. To ensure that rhyme would be attended to, the subjects were told to expect pairs of rhymes. The rhyme pairs were members of small, medium, or large rhyme sets and all pairs were semantically unrelated, as in CLOCK BLOCK. Recall was cued with the context words or with extralist rhymes or extralist associates of the targets. Given that elaborative semantic encodings were formed, the question was whether access would be primarily limited to a single domain of encoded information, regardless of the information available in the test cue, or whether all domains would be accessed.

Access to the rhyme domain is demonstrated by recall that varies as a function of the rhyme set size of the pairs. If rhyme is accessed, larger rhyme sets will be associated with lower levels of recall (e.g., Nelson, Bajo, \& Canas, 1987). Access to encoded meaning is demonstrated by better recall with imagery instructions than with repetition instructions (Bower \& Winzenz, 1970). Presumably, if access is selective and can be confined to one domain or the other, the effects of manipulations in the contrasting domain will be reduced or eliminated. If access is primarily confined to rhyme, rhyme set-size effects should be comparable for each instructional set and the normal advantage of imagery instructions will be less evident. Alternatively, if access is primarily confined to meaning, rhyme set-size effects will be less apparent and imagery instruc- 
tions will differentially facilitate recall. Finally, if access is not selective, both rhyme and instructional effects should be equally evident for all three types of test cues.

In Experiment 2 rhyme set size and encoding instructions were also varied, but only study-context words were used as test cues. Amount of encoding time was manipulated in an attempt to vary the quality of the semantic encoding, and pronunciation of the rhyme test cue was manipulated in an attempt to vary attention to word sound at test.

\section{EXPERIMENT 1}

\section{Method}

Design and Subjects. The experimental design formed a $2 \times 3 \times 3$ mixed model factorial. Instructions (interactive imagery/repetition) and type of test cue (study/rhyme/associate) were manipulated between subjects, with rhyme set size (small/medium/large) varied within subjects. The subjects were students in introductory psychology classes, selected under an incentive system. Twelve subjects were assigned to each between-subjects condition, with 6 assigned to each of two lists, so that a total of 72 served in the experiment. They were randomly assigned to conditions in replication blocks of 12 subjects.

Materials. Controlled association norms for rhyme were obtained for over 700 words from introductory psychology classes ranging in size from 30 to 245 students. The students were given booklets containing words printed in uppercase letters with a blank next to each word. They were asked to write the first word they thought of that rhymed with the word on the left. Single rather than multiple responses were used to avoid problems with response chaining and retrieval inhibition, and because the first response appears to provide a better estimate of set size (Joelson \& Herrmann, 1978).

These norms were used to estimate rhyme set size by counting the total number of different but appropriate rhymes given for each cue, and relative strength of the responses was estimated by the proportion of students who gave each particular response. With these estimates in mind, two 27-pair lists were constructed for presentation during the study phase. The study cues for these lists had served as the rhyme cues in the norms, and the targets were taken from the rhyme responses provided by the students. These lists are shown in Table 1. Each list was subdivided into three sets of 9 pairs so that the study cue defined rhyme sets that were small, medium, or large. Small sets averaged $6.67(S D=1.97)$ rhymes, medium sets averaged $11.50(S D=.92)$ rhymes, and large sets averaged 18.17 $(S D=2.60)$ rhymes. The relative strength between the study cues and their rhyming targets was held constant over conditions of set size and averaged $11(S D=.08)$. For example, LEG defined a small set of 6 other rhyming words, one of which was KEG, which was provided by $12 \%$ of the subject sample, CAPE defined a mediumsized set of 12 rhymes, one of which was TAPE, which was also given by $12 \%$ of the subjects, and so forth. Finally, all words appearing in the study list were concrete and averaged $5.70(S D=.52)$ on the Toglia and Battig (1978) scale.

Two extralist cues were selected for each target; these cues are also shown in Table 1 . The extralist rhyme cues were taken from the rhyme norms. As would be expected, their set sizes covaried with the rhyme set sizes of their targets, showing values of 6.69 $(S D=2.30), 10.50(S D=2.80)$, and $15.44(S D=4.11)$ for small, medium, and large rhyme sets, respectively. As with the study cues, the strength of the relationship between the extralist cues and their targets was equated in each condition of set size, averaging .11

Table 1

Materials Used in Experiment 1

\begin{tabular}{|c|c|c|c|c|c|c|c|}
\hline \multicolumn{4}{|c|}{ List 1} & \multicolumn{4}{|c|}{ List 2} \\
\hline \multicolumn{2}{|c|}{ Study Pairs } & \multicolumn{2}{|c|}{ Extralist Cues } & \multicolumn{2}{|c|}{ Study Pairs } & \multicolumn{2}{|c|}{ Extralist Cues } \\
\hline Cue & Target & Rhyme & Associate & Cue & Target & Rhyme & Associate \\
\hline LEG & KEG & BEG & BARREL & BENCH & TRENCH & WRENCH & DITCH \\
\hline BATH & PATH & MATH & TRAIL & CARD & GUARD & HARD & SENTRY \\
\hline GHOST & TOAST & POST & JELLY & LEG & KEG & BEG & BEER \\
\hline HOOD & WOOD & GOOD & LOG & GHOST & POST & HOST & POLE \\
\hline PELT & BELT & FELT & WAIST & GLOVE & DOVE & SHOVE & PIGEON \\
\hline ROBE & GLOBE & PROBE & WORLD & HOUSE & BLOUSE & SPOUSE & SHIRT \\
\hline STOVE & GROVE & CLOVE & ORCHARD & LIVER & RIVER & SHIVER & BRIDGE \\
\hline WRENCH & TRENCH & BENCH & DITCH & MOOSE & CABOOSE & NOOSE & TRAIN \\
\hline GIRAFFE & CALF & STAFF & cow & BEEF & LEAF & CHIEF & TREE \\
\hline CAPE & TAPE & GRAPE & GLUE & BOOT & FLUTE & SUIT & CLARINET \\
\hline FOG & DOG & LOG & FLEA & CAT & HAT & BRAT & HELMET \\
\hline DUCK & TRUCK & TUCK & CAR & CORD & SWORD & BOARD & KNIGHT \\
\hline FILE & SMILE & BILE & FROWN & LAMP & STAMP & CAMP & MAIL \\
\hline BUN & SUN & RUN & $\mathrm{BEACH}$ & LID & SQUID & KID & OCTOPUS \\
\hline HOOK & BOOK & TOOK & MAGAZINE & NECK & CHECK & PECK & MONEY \\
\hline PEN & WREN & DEN & SPARROW & PEN & HEN & DEN & ROOSTER \\
\hline BOOT & FLUTE & SUIT & ORCHESTRA & CAPE & GRAPE & TAPE & RAISIN \\
\hline PARK & SHARK & BARK & BARRACUDA & HOP & MOP & TOP & FLOOR \\
\hline CLOCK & BLOCK & SHOCK & BRICK & CAVE & WAVE & SLAVE & OCEAN \\
\hline RAKE & SNAKE & LAKE & WORM & CLOCK & ROCK & SHOCK & PEBBLE \\
\hline PHONE & CONE & BONE & CYLINDER & CONE & BONE & PHONE & SPINE \\
\hline PILL & DRILL & STILL & DENTIST & KEY & $\mathrm{BEE}$ & TEA & HONEY \\
\hline PLATE & GATE & LATE & FENCE & SKATE & CRATE & PLATE & BOX \\
\hline TIE & PIE & LIE & CAKE & CHAIR & BEAR & HAIR & LION \\
\hline CAVE & PAVE & SLAVE & ROAD & PILL & DRILL & STILL & DENTIST \\
\hline SAW & LAW & JAW & POLICE & LAKE & CAKE & RAKE & MUFFIN \\
\hline MOON & SPOON & RACCOON & FORK & DRUM & GUM & HUM & BUBBLE \\
\hline
\end{tabular}

Note-The first 9 study pairs in each list have small rhyme sets, the next 9 have medium-sized sets, and the last 9 have large rhyme sets. 
$(S D=.09)$. The extralist meaning cues were taken from associative meaning norms in which subjects were asked to produce the first word that came to mind that was meaningfully related to the presented word (see Nelson \& McEvoy, 1979, for details). The strength of the relationship between these cues and the targets shown in Table 1 was equated in each condition of rhyme set size and averaged $.14(S D=.13)$ over all conditions.

Procedure. The subjects participated in individual sessions and each received a single study-test trial. The rhyme pairs presented for study were typed in uppercase letters, with the study cue presented to the left and underlined; all pairs were presented by a Kodak Carousel slide projector driven at a 4-sec rate. The subjects were required to read each pair aloud when it appeared on the screen.

All subjects were told that their task was to learn what rhymes went together. Rhyme was mentioned to ensure that this dimension would be attended. Interactive-imagery subjects were told that the most efficient way to learn the pairs was to construct a mental picture representing the two words interacting together in some way. Repetition subjects were told that greatest efficiency would be achieved by repeating the words aloud as often as possible until the next pair appeared. Examples and illustrations were provided to both groups of subjects; for the pair JET NET, for example, the imagery subjects were told to imagine a jet stuck in a net and the repetition subjects were told to repeat "jet net" several times. All subjects were given a short practice list on which they were tested. Imagery subjects were then shown the practice pairs again and were asked how they imagined each one. At this point, the experimenter discouraged the use of other strategies, such as separation imagery, and encouraged the development of interacting images. This second presentation of the practice pairs was only for instructional purposes, and did not occur with the actual experimental pairs. After this instructional session the experimental list was shown.

Immediately after the last study pair was shown, the test instructions were read. In the study-cue condition, the subjects were told to expect the left-hand word from each pair as a cue for the righthand word. In the extralist-cue conditions, they were told to recall words that appeared on the right side of the screen and that some new words (rhymes or meaning-related associates) would be presented to help them remember each word. Examples were provided to ensure that the subjects understood the instructions and the nature of the relationship between the cues and the words to be recalled.

All test cues appeared in uppercase letters, were underlined, and had to be pronounced aloud when shown. Each cue was presented at a subject-paced rate so that the subjects had as much time as needed to produce the study word. Finally, the orders of presentation of both study pairs and test cues were independently and unsystematically randomized for each subject.

\section{Results and Discussion}

Table 2 shows the probability of correct recall as a function of study trial instructions, type of test cue, and rhyme set size. As can be seen, imagery instructions produced higher recall than repetition instructions, original cues were superior to extralist cues, and rhyme set-size effects were apparent in all conditions. A three-factor analysis of variance indicated that the effects of instructions $\left[F(1,66)=12.26, M S_{\mathrm{e}}=.057\right]$, type of test cue $[F(2,66)=31.50]$, and rhyme set size $[F(1,66)=$ $\left.6.07, M S_{e}=0.26\right]$ were all significant sources of variance (the .05 criterion was used). The probability of correct recall was .54 after imagery instructions and .42 after repetition instructions. A Fisher's two-tailed least significant difference $(L S D)$ of .08 indicated that recall was higher for original study cues (.66) than for extralist
Table 2

Probability of Correct Recall as a Function of Study Trial Instructions, Type of Test Cue, and Rhyme Set Size

\begin{tabular}{llccc}
\hline \multirow{2}{*}{$\begin{array}{c}\text { Test } \\
\text { Cue }\end{array}$} & Study Trial & \multicolumn{3}{c}{ Rhyme Set Size } \\
\cline { 3 - 5 } Study & Instructions & Small & Medium & Large \\
\hline \multirow{3}{*}{ Rhyme } & Imagery & .79 & .69 & .69 \\
& Repetition & .66 & .55 & .57 \\
Associative & Imagery & .52 & .49 & .43 \\
& Repetition & .42 & .35 & .29 \\
& Imagery & .47 & .37 & .37 \\
& Repetition & .33 & .31 & .27 \\
\hline
\end{tabular}

rhymes (.42) and extralist associates (.35), which did not differ reliably. Finally, recall systematically decreased as rhyme set size increased from small (.53) to medium (.46) to large (.44). A Fisher's $L S D$ of .05 indicated that recall for both medium and large sets was significantly less than recall for small sets. No other sources in this analysis even approached the criterion for significance, with all $F$ s less than unity.

These results indicate that retrieval access was not restricted to either the rhyme domain or the meaning domain. Rhyme set-size effects were apparent under both instructional conditions and instructional effects were apparent within each level of rhyme set size. Most importantly, both rhyme set-size and instructional effects were independent of the type of information represented in the test cue. Comparable set-size and instructional effects were obtained for the original study-context words, for extralist rhymes, and for extralist associates. The original study-context word did not have to be present at test to retrieve encoded rhyme information or to instantiate the imaginal encoding. Rhyme encodings resulting from activating related rhymes were accessed even when testing was prompted with extralist meaning cues. Similarly, semantic encodings resulting from interactive imagery were accessed even when testing was prompted by extralist rhyme cues. These results, in conjunction with previous results, suggest that semantic encodings are accessed by rhyme cues when these encodings are the result of elaborative processes, such as interactive imagery, and when they are the result of automatic processes, such as those associated with meaning set-size effects (Nelson, Bajo, \& Canas, 1987). These findings may help to explain why testing effects are obtained even when cues from different domains are used on the first and second tests. McDaniel and Masson (1985) gave subjects an initial cued recall test with either thyme or semantic cues, then a second test with cues from either the same or the alternative domain. Testing effects obtained when the domains were switched may have occurred because cues from one domain provide access to information in both domains, not just the domain indicated in the test cue.

The primary implication of these findings is that, in the cued recall task, information encoded in both domains is accessed regardless of the domain of information specified in the cue. If access could have been more selective, set-size and instructional effects should have been more 
specific to the information directly available in the test cue. It is particularly interesting that the presentation of the original study-context words as test cues produces rhyme set-size effects even after they have been incorporated into imaginal encodings. Although these cues rhyme with their targets, it seems reasonable to assume that imaginal elaboration at study would have been sufficient to allow selective access to the imaginal representations at test. The purpose of Experiment 2 was to explore this finding in more detail.

\section{EXPERIMENT 2}

Experiment 2 was designed to replicate and extend the portion of the initial experiment involving the use of studycontext rhymes as test cues. Intralist cues are more interesting than extralist cues in this situation because they are directly involved during the study-trial encoding and offer the option of selective access to encoded rhyme or meaning or to both types of information at test. As in the first experiment, rhyme pairs defining small, medium, or large sets were presented under either repetition or imagery instructions. In addition, amount of available study time and test-cue pronunciation requirements were varied in an attempt to isolate a set of conditions that, if possible, would produce selective access to meaningfully encoded information. The pairs were presented for 3 or $5 \mathrm{sec}$, on the assumption that more time would allow for the creation of more effective semantic encodings (Bugelski, Kidd, \& Segmen, 1968). The requirement to pronounce aloud the rhyming test cues was varied on the assumption that this manipulation would vary attention to the phonemic domain during the test trial. Half of the subjects were required to name the test cues aloud, as in the initial experiment, and half were told to use the cues to recall the targets, without mentioning anything about naming them. The rationale underlying the naming manipulation was that the act of naming the cue would direct attention to word sound, which would instantiate the rhyme encoding (cf. Nelson, Bajo, \& Canas, 1987). Without such a requirement, selective access to what has been meaningfully encoded may be possible, at least under some conditions of encoding. For example, with imagery instructions, with a longer study period, or with both conditions in force, information encoded within the rhyme domain may be effectively bypassed so long as no attention is drawn to word sound during testing. Alternatively, if selective access to information encoded in both phonemic and meaning domains is impossible, then rhyme and instructional effects should be equally apparent under all conditions, as was the case in the first experiment.

\section{Method}

Design. The design formed a $2 \times 2 \times 2 \times 3$ factorial with instructions, presentation rate, and pronunciation requirement manipulated between subjects and rhyme set size varied within subjects. Six- teen subjects were assigned to each between-subjects condition, with 8 assigned to each of two lists. The subjects came from the same source as used in the initial experiment and were assigned to conditions in a similar manner.

Materials. The rhyme pairs used to construct the two lists for this experiment were taken from the norms used for Experiment 1; because $47 \%$ of the pairs were new in the present experiment, the lists are presented in full in Table 3 . To avoid ceiling effects at the slower rate, the study list was lengthened by $25 \%$ relative to the list used in Experiment 1. The average rhyme set size was 6.46 $(S D=1.91), 10.88(S D=1.45)$, and $18.63(S D=2.45)$ for small, medium, and large rhyme sets, respectively. The normative strength of association between the rhyme cues and their targets was equated at each level of set size and averaged $.11(S D=.07)$. Furthermore, concreteness ratings were equated for cues and targets for each set size, with an overall average rating of $5.69(S D=.43)$. Thus, all items were high in rated concreteness.

Procedure. The general procedural details were identical to those used in Experiment 1. However, the presentation of the rhyme pairs during study was paced at either 3 or $5 \mathrm{sec}$ per pair, and either the test cues were named prior to target recall or the instructions never mentioned naming.

Table 3

Materials Used in Experiment 2

\begin{tabular}{|c|c|c|c|}
\hline \multicolumn{2}{|c|}{ List 1} & \multicolumn{2}{|c|}{ List 2} \\
\hline Cue & Target & Cue & Target \\
\hline BATH & PATH & BENCH & TRENCH \\
\hline BEEF & LEAF & CARD & GUARD \\
\hline FORK & STORK & EGG & KEG \\
\hline GHOST & TOAST & FORK & CORK \\
\hline GIRAFFE & CALF & GHOST & POST \\
\hline HARP & TARP & GLOVE & DOVE \\
\hline HOOD & WOOD & HOUSE & BLOUSE \\
\hline LEG & KEG & LIVER & RIVER \\
\hline PELT & BELT & MASK & FLASK \\
\hline ROBE & GLOBE & MOOSE & CABOOSE \\
\hline STOVE & GROVE & STEM & GEM \\
\hline WRENCH & TRENCH & TEETH & WREATH \\
\hline BOAT & GOAT & BOOT & FLUTE \\
\hline CAPE & TAPE & CAPE & GRAPE \\
\hline CART & HEART & CAT & HAT \\
\hline CORD & BOARD & CORD & SWORD \\
\hline DUCK & TRUCK & FOG & LOG \\
\hline FILE & SMILE & GUN & BUN \\
\hline HAND & LAND & LAMP & STAMP \\
\hline HOOK & COOK & LID & SQUID \\
\hline LAMP & CLAMP & MAN & PAN \\
\hline NECK & DECK & PARK & SHARK \\
\hline PEACH & BLEACH & PEN & HEN \\
\hline PEN & WREN & PIG & WIG \\
\hline CAVE & PAVE & BRICK & TICK \\
\hline CHAIR & BEAR & CAVE & WAVE \\
\hline CLOCK & BLOCK & CLOCK & ROCK \\
\hline DRUM & GUM & CONE & BONE \\
\hline HEAD & SHED & DRUM & PLUM \\
\hline KEY & TEA & HEAD & BREAD \\
\hline LAKE & CAKE & KEY & BEE \\
\hline MOON & SPOON & KING & SPRING \\
\hline PHONE & CONE & PILL & HILL \\
\hline PILL & DRILL & PLANE & CANE \\
\hline PLATE & GATE & PLATE & CRATE \\
\hline TIE & PIE & SAW & PAW \\
\hline
\end{tabular}

Note--The first 12 study pairs in each list have small rhyme sets, the next 12 have medium-sized sets, and the last 12 have large rhyme sets. 


\section{Results and Discussion}

Table 4 displays the probabilities of correct recall for the principal conditions. Examination of these values indicated that higher recall levels were attained for imagery instructions, for the slower rate of presentation, and for the testing condition in which the test cues were not pronounced aloud. Although rhyme set-size effects were apparent in every condition, they appeared to be reduced at the slow presentation rate when test cues were not named. A four-factor analysis of variance indicated that the effects of instructions $\left[F(1,120)=48.47, M S_{\mathrm{e}}=\right.$ $.084]$, presentation rate $[F(1,120)=19.25]$, pronunciation $[F(1,120)=4.69]$, and rhyme set size $[F(2,120)=$ $\left.37.39, M S_{\mathrm{e}}=.015\right]$ were all significant sources. The probabilities of correct recall were .66 and .45 with imagery and repetition instructions, respectively; .62 for the slow presentation rate and .49 for the fast rate; and .59 when test cues were not named and .53 when they were named. Finally, recall systematically decreased as rhyme set size increased from small (.63) to medium (.54) to large (.50).

Table 4 also shows that the rhyme set-size effect was attenuated under imagery instructions, relative to repetition instructions. This interaction is displayed in the bottom row of the table. The results of the statistical analysis indicated that this interaction was reliable $[F(2,120)=$ 3.62]. However, a Fisher's $L S D$ of .04 showed that significant rhyme set-size effects were apparent under each instructional condition, indicating that the interaction reflected attenuation, but not elimination, of the set-size effect. The only other interesting trend was apparent when presentation rate was slow and pronunciation was not required. The rhyme set-size effect was reduced under both imagery and repetition instructions in this condition. This reduction was reflected in the only remaining significant source in the analysis, the rate $\times$ pronunciation $\times$ setsize interaction $[F(2,120)=4.65]$. When pooled over instructions, the probabilities of recall for small, medium, and large rhyme sets were, respectively, .67, .64, and .64 for the slow rate/no pronunciation condition. By a Fisher's $L S D$ of .06 , none of those differences was reliable. This contrasts with significant set-size effects in all the remaining conditions involving rate and pronunciation.

The results of this experiment are consistent with those of Experiment 1 in that access in most conditions was not limited to either the rhyme or meaning domain. Rhyme set-size effects generally were apparent in each condition of instruction, and instructional effects were apparent within each level of rhyme set size. This finding indicates that the subjects tended to access information encoded in both domains. However, they apparently bypassed the rhyme domain under one set of conditions. Regardless of encoding instructions, relatively long study periods coupled with absence of the naming requirement substantially reduced the rhyme set-size effect. Only with these encoding/retrieval conditions was access apparently confined to the meaning domain. The longer study period permits the development of superior semantic encodings, but even with such encodings, access is confined to meaning only when the test cue does not have to be named. Naming draws attention to word sound and this requirement is apparently sufficient to instantiate the rhyme encoding. When coupled with previous results (Nelson, Bajo, \& Canas, 1987), this finding suggests that information encoded about word sound can be bypassed in the cued recall task, but only under conditions that do not draw attention to that domain during testing.

Because of the theoretical importance of the null effect of rhyme set size, an attempt was made to replicate the finding on another sample of 16 subjects. The rhyme pairs shown in Table 3 were presented at the slower 5-sec rate during study and the subjects were given the interactive imagery instructions and were not required to name the test cues. In addition, postexperiment interviews were conducted to determine whether encoding strategy varied with rhyme set size. It was possible, although unlikely, that the subjects used imagery more frequently for pairs with larger sets; if this were the case, the elimination of the rhyme set-size effect would be due to differences in encoding, not to differences in selective access. After the test trial, each pair was shown individually and the subjects were asked to describe how they had learned it. Although such verbal reports have obvious shortcomings, these shortcomings apply equally to each condition of set size.

The results of this follow-up study replicated the findings from the main experiment. The probabilities of correct recall for small, medium, and large rhyme sets were, respectively, $.80, .74$, and .78 , and these values were not reliably different $\left[F(2,30)=1.15, M S_{\mathrm{e}}=.010\right]$. Table 5 shows the results of the postexperiment interviews. Each subject's description of how each pair had been learned was categorized as involving interactive imagery (e.g., "I imagined a LEG kicking an empty beer KEG"), repetition (e.g., "I just repeated it"), or other (e.g., "BOAT GOAT reminded me of Noah's ark"). The values in

Table 4

Probability of Correct Recall as a Function of Encoding Instructions, Presentation Rate, Naming Requirements, and Rhyme Set Size

\begin{tabular}{lcccccccc}
\hline & & \multicolumn{3}{c}{ Imagery } & & \multicolumn{3}{c}{ Repetition } \\
\cline { 3 - 5 } \cline { 7 - 8 } Rate & Naming & Small & Medium & Large & & Small & Medium & Large \\
\hline Fast & Yes & .58 & .50 & .53 & & .47 & .39 & .25 \\
& No & .71 & .59 & .59 & & .54 & .40 & .34 \\
Slow & Yes & .80 & .66 & .63 & & .59 & .51 & .38 \\
& No & .79 & .77 & .76 & & .55 & .51 & .52 \\
Mean & & .72 & .63 & .63 & & .54 & .45 & .37 \\
\hline
\end{tabular}


Table 5

Probability of Correct Recall Based on Reported Strategies

\begin{tabular}{lccc}
\hline $\begin{array}{l}\text { Reported } \\
\text { Strategy }\end{array}$ & \multicolumn{3}{c}{ Rhyme Set Size } \\
\hline Small & Medium & Large \\
\hline Imagery & $.83(.79)$ & $.78(.83)$ & $.82(.80)$ \\
Repetition & $.56(.16)$ & $.39(.12)$ & $.42(.14)$ \\
Other & $1.00(.05)$ & $1.00(.06)$ & $1.00(.06)$ \\
\hline
\end{tabular}

Note-Values in parentheses reflect the proportion of times the strategy was used.

parentheses show the proportion of times the reported strategy was used, and, as can be seen, imagery was used with approximately equal frequency at each level of rhyme set size $(.79, .83$, and .82 for small, medium, and large rhyme sets, respectively). In addition, given that imagery was reportedly used, the probability of correct recall did not vary with set size. These data indicate that reported use of the imagery strategy did not vary across rhyme set size, and when it was used, it was as effective for pairs with large sets as it was for pairs with smaller sets. Hence, given an effective semantic encoding and the absence of a naming requirement at test, information encoded in the rhyme domain apparently can be bypassed.

\section{GENERAL DISCUSSION}

The results of these experiments demonstrate two main points about the probable neighborhoods of information accessed during cued recall. The first and most important point is that access can be selective, at least under some conditions. Access can be limited to a single domain, even though information has been encoded within two domains. Access was limited to encoded meaning when the longer study period was combined with the elimination of the requirement to name the test cues aloud. When both of these contingencies were in effect, rhyme set size failed to have a reliable effect. When encoding spanned both meaning and rhyme domains, selective access that bypasses the rhyme domain was feasible. However, in no case did the results indicate that access was ever restricted to the rhyme domain. Instructional effects were evident in every comparison in both experiments. Even when recall was cued by extralist rhymes that had to be named, the relative advantage of imagery instructions was still evident. At least in the cued recall task, information encoded about meaning is not likely to be bypassed even when the conditions of testing strongly favor the rhyme domain. Hence, selective access to encoded meaning appears to be critical to performance in this task.

The second point is that with domains of information as different as word sound and word meaning, access to what has been encoded is not limited by the information directly available in the test cue. Given that information has been encoded within two domains, both domains are generally accessed at test regardless of the domain of information directly available in the test cue. In these experiments, information encoded about rhyme and mean- ing was accessed regardless of whether that access was initiated by study-context words, by extralist rhymes, or by extralist meaning cues. Thus, consistent with the encoding specificity principle (Fisher \& Craik, 1977; Tulving \& Thomson, 1973), it appears that retrieval operations are not driven exclusively by information that is directly represented in the retrieval cue. This information serves only to initiate the process and, once it is initiated, the domains of encoding tend to determine the domains of information that are accessed at test. Thus, thyme cues provide access to encoded meaning, regardless of whether that encoding is the result of automatic activation processes or directed encoding strategies such as interactive imagery. Similarly, meaning cues provide access to information encoded about rhyme. Rhyme set-size effects were apparent with extralist meaning cues even after the pairings were interactively encoded. These effects were also apparent with the study-context cues, provided that they were named during testing. In general, the domains of encoding controlled the domains of information accessed at test.

The present findings do not allow determination of which domain is accessed initially when information has been encoded within two domains. However, the sensorysemantic model suggests a possible sequence of events in the cued recall task (Nelson, 1981, 1989). When test cues provide information concerning only a single domain, as when extralist rhyme or meaning cues are provided, access is initiated in that domain. The entries recovered in this phase of retrieval are presumed to be unidimensional, consisting of only phonemic or semantic entries. These entries serve as internally generated retrieval cues that access episodically encoded information, including information encoded in the other domain. A phonemic entry may serve as a cue for recovering encoded meaning, and an associatively related entry may serve to recover information encoded about word sound. When the test cue can be used to specify either domain of encoding, as when study-context words are used as test cues, either domain can be accessed initially. Which domain is accessed first depends on the relative salience of the information provided for each domain by the cue and other contingencies operating during testing. For example, as in Experiment 2, when semantic information was more salient because of long study times and rhyme information was less salient at test because the cues were not named, the semantic domain was apparently accessed first and the rhyme domain was apparently not accessed at all.

The fact that selective retrieval access can occur, even if only under highly restricted conditions, suggests that access from one domain of encoding to another in the cued recall task is not completely automatic, as has been assumed (e.g., Nelson, 1989). In the present context the term automatic implies that encoded information in all domains should invariably be accessed. Set-size and instructional effects should have been apparent without exception across variations in study conditions, type of test cue, and other testing requirements such as naming the cue. 
However, the results of this study suggest that selective retrieval access is possible. Subjects may attend to one domain of encoded information or both, just as they follow instructions to rehearse or to develop interactive images. In this sense, subjects may select the domain to be searched, with the choice presumably dictated by knowledge of what has been encoded, and therefore of what kind of information is likely to prove useful in meeting task criteria. Once attention is directed toward a particular domain, relevant information within that domain is accessed. Hence, when subjects are given a relatively long study period to encode each rhyme pairing, they presumably attempt to develop effective semantic encodings because of habitual tendencies to focus on meaning. When given the context words as the test cues, they attempt to access these semantic encodings in order to meet task demands. Normally, access to the rhyme domain can be, and apparently is, bypassed, unless the test instructions require the naming of each cue. This requirement directs attention to the sound of the cue, thus providing access to information encoded in the phonemic domain.

In this view, retrieval involves selective access that is determined by what has been encoded and by other aspects of the testing context. Other research has shown that encoded meaning, although normally accessed in cued recall, is not normally accessed in fragment completion, even when instructions explicitly mention the study encoding (Graf \& Mandler, 1984; Nelson, Canas, Bajo, \& Keelean, 1987; Roediger \& Blaxton, 1987). In fragment completion, the test cue and task requirements emphasize the production of a word as a lexical unit that fits the fragment cue, and encoded meaning is not accessed. Thus, information encoded about meaning can be bypassed, just as information encoded about rhyme can be bypassed. Retrieval appears to involve selective components that are guided by the information directly available in the cue, but retrieval is by no means limited to this information. What has been encoded about the target experience itself plays an important, if not a crucial, role in determining what domains are accessed during retrieval.

\section{REFERENCES}

ANDERSON, J. R. (1983). A spreading activation theory of memory. Journal of Verbal Learning \& Verbal Behavior, 22, 261-295.

BaHrick, H. P. (1970). Two-phase model for prompted recall. Psychological Review, 77, 215-222.

BOWER, G. H., \& WINZENZ, D. (1970). Comparison of associative leaming strategies. Psychonomic Science, 20, 119-120.

BUGELSKI, B. R., KIDD, E., \& SEGMEN, J. (1968). Image as a mediator in one-trial paired-associate learning. Journal of Experimental Psychology, 76, 69-73.
Fisher, R. P., \& Craik, F. I. M. (1977). Interaction between encoding and retrieval operations in cued recall. Joumal of Experimental Psychology: Human Learning \& Memory, 3, 701-711.

GraF, P., \& MANDLER, G. (1984). Activation makes words more accessible, but not necessarily more retrievable. Joumal of Verbal Learming \& Verbal Behavior, 23, 553-568.

James, W. (1962). Psychology: Briefer course. New York: Collier Books. (Original work published 1890)

Joelson, J. M., \& Herrmann, D. J. (1978). Properties of categories in semantic memory. American Journal of Psychology, 91, 101-114.

McDaniel, M. A., \& Masson, M. E. J. (1985). Altering memory representations through retrieval. Journal of Experimental Psychology: Learning, Memory, \& Cognition, 11, 371-385.

Nelson, D. L. (1981). Many are called but few are chosen: The influence of context on the effects of category size. In G. H. Bower (Ed.), The psychology of learning and motivation, 15. New York: Academic Press.

NeLSON, D. L. (1989). Implicitly activated knowledge and memory. In C. Izawa (Ed.), Current issues in cognitive psychology: The Tulane Flowerree Symposium on Cognition. New York: Erlbaum.

Nelson, D. L., Bajo, M. T., \& Canas, J. (1987). Prior knowledge and memory: The episodic encoding of implicitly activated associates and thymes. Journal of Experimental Psychology: Learning. Memory, \& Cognition, 13, 54-63.

Nelson, D. L., Canas, J., Bajo, M. T., \& Keelean, P. (1987). Comparing word fragment completion and cued recall with letter cues. Journal of Experimental Psychology: Learning, Memory, \& Cognition, 13, 542-552.

Nelson, D. L., \& Friedrich, M. A. (1980). Encoding and cuing sounds and senses. Journal of Experimental Psychology: Human Learning \& Memory, 6, 717-731.

Nelson, D. L., \& McEvoy, C. L. (1979). Encoding context and set size. Joumal of Experimental Psychology: Human Learning \& Memory, 5, 292-314.

Nelson, D. L., McEvoy, C. L., \& Friedrich, M. A. (1982). Extralist cuing and retrieval inhibition. Journal of Experimental Psychology: Learning, Memory, \& Cognition, 8, 89-105.

Nelson, D. L., Walling, J. R., McEvoy, C. L. (1979). Doubts about depth. Joumal of Experimental Psychology: Human Learning \& Memory, 5, 24-44.

RaAijmakers, J. G., \& ShIFFrin, R. M. (1981). Search of associative memory. Psychological Review, 88, 93-134.

RoEDIGER, H. L., III, \& BLAXTON, T. A. (1987). Retrieval modes produce dissociations in memory for surface information. In D. S. Gorfein \& R. R. Hoffman (Eds.), Memory and cognitive processes: The Ebbinghaus Centennial Conference (pp. 349-379). Hillsdale, NJ: Erlbaum.

Shiffrin, R. M. (1970). Memory search. In D. A. Norman (Ed.), Models of human memory. New York: Academic Press.

TogLiA, M. P., \& BATTIG, W. F. (1978). Handbook of semantic word norms. Hillsdale, NJ: Erlbaum.

Tulving, E., \& Thomson, D. M. (1973). Encoding specificity and retrieval processes in episodic memory. Psychological Review, 80, $352-372$.

(Manuscript received October 27, 1988; revision accepted for publication April 28, 1989.) 